\title{
Exciton dephasing in ZnSe quantum wires
}

Wagner, Hans Peter; Langbein, Wolfgang Werner; Hvam, Jørn Märcher; Bacher, G.; Kümmell, T.; Forchel, A.

Published in:

Physical Review B

Link to article, DOI:

10.1103/PhysRevB.57.1797

Publication date:

1998

Document Version

Publisher's PDF, also known as Version of record

Link back to DTU Orbit

Citation (APA):

Wagner, H. P., Langbein, W. W., Hvam, J. M., Bacher, G., Kümmell, T., \& Forchel, A. (1998). Exciton dephasing in ZnSe quantum wires. Physical Review B, 57(3), 1797-1800. https://doi.org/10.1103/PhysRevB.57.1797

\section{General rights}

Copyright and moral rights for the publications made accessible in the public portal are retained by the authors and/or other copyright owners and it is a condition of accessing publications that users recognise and abide by the legal requirements associated with these rights.

- Users may download and print one copy of any publication from the public portal for the purpose of private study or research.

- You may not further distribute the material or use it for any profit-making activity or commercial gain

- You may freely distribute the URL identifying the publication in the public portal

If you believe that this document breaches copyright please contact us providing details, and we will remove access to the work immediately and investigate your claim 


\title{
Exciton dephasing in ZnSe quantum wires
}

\author{
H. P. Wagner \\ Universität Regensburg, Institut Physik II, D-93040 Regensburg, Germany \\ W. Langbein and J. M. Hvam \\ Mikroelektronik Centret, The Technical University of Denmark, 2800 Lyngby, Denmark \\ G. Bacher, T. Kümmell, and A. Forchel \\ Technische Physik, Universität Würzburg, Am Hubland, D-97074 Würzburg, Germany
}

(Received 10 July 1997)

\begin{abstract}
The homogeneous linewidths of excitons in wet-etched $\mathrm{ZnSe}$ quantum wires of lateral sizes down to $23 \mathrm{~nm}$ are studied by transient four-wave mixing. The low-density dephasing time is found to increase with decreasing wire width. This is attributed mainly to a reduction of electron-exciton scattering within the wire due to the electron trapping in surface states and exciton localization. The exciton-exciton scattering efficiency, determined by the density dependence of the exciton dephasing, is found to increase with decreasing wire width. This is assigned to the reduced phase space in a quasi-one-dimensional system, enhancing the repulsive interaction between excitons due to Pauli blocking.
\end{abstract}

[S0163-1829(98)00503-7]

Ultrafast degenerate four-wave mixing (FWM) techniques have been successfully applied to study the fundamental coherent processes in bulk and quasi-two-dimensional (2D) structures of both III-V (Refs. 1-7) and II-VI compounds ${ }^{8-13}$ over the last decade. The investigation of the coherent phenomena in quantum wires (QWR's) has recently become possible due to major improvements in patterning technology and growth techniques. This development is driven by the supposed superiority of QWR's for application in optoelectronic devices, where improvements as the reduction of laser threshold or the decrease of scattering processes are expected due to the modified density of states. ${ }^{14-18}$

Until recently, the efforts to fabricate low-dimensional structures by lateral patterning have focused on III-V semiconductors, ${ }^{19-21}$ for which degenerate FWM experiments are reported on reactive-ion etched structures. ${ }^{22} \mathrm{Fol}-$ lowing the current search for blue-green laser structures based on $\mathrm{ZnSe}$ and $\mathrm{Zn}_{x} \mathrm{Cd}_{1-x}$ Se quantum wells (QW's), high-quality II-VI structures are available for quantum wire and dot fabrication. ${ }^{23,24}$ Since the wide-gap II-VI materials have in general a much larger exciton oscillator strength and thus a higher third-order nonlinearity than III-V materials, ${ }^{25}$ they are better suited for the study of coherent transient phenomena in QWR's, which in general suffer by a small active volume.

In this paper, we report on subpicosecond FWM studies on QWR's prepared from a single $\mathrm{ZnSe} / \mathrm{Zn}_{1-x} \mathrm{Mg}_{x} \mathrm{Se} \mathrm{QW}$ (SQW), thus avoiding propagation effects. ${ }^{26,27}$ These investigations are possible due to the high excitonic oscillator strength in $\mathrm{ZnSe}$. We investigate the low-density dephasing rate and the exciton-exciton scattering rate by densitydependent FWM on QWR's of lateral sizes down to $23 \mathrm{~nm}$.

The QWR structures have been processed by electronbeam lithography and wet chemical etching from a $\mathrm{Zn}_{0.94} \mathrm{Mg}_{0.06} \mathrm{Se} / \mathrm{ZnSe} \mathrm{SQW}$ sample, which was pseudomorphically grown on (001) GaAs by molecular-beam epitaxy. ${ }^{28}$
The active ZnSe layer of $16 \mathrm{~nm}$ thickness is sandwiched between two $25 \mathrm{~nm}$-thick $\mathrm{Zn}_{0.94} \mathrm{Mg}_{0.06}$ Se barriers, defining a type-I QW. ${ }^{13,29}$ The patterned wire arrays have sizes of 100 $\times 100 \mu \mathrm{m}^{2}$ and comprise wires from 500 down to $23 \mathrm{~nm}$ width at an area filling factor of 0.05-0.3. By aligning the wires in the $\langle 011\rangle$ crystal orientation, steep sidewalls and smooth surfaces in the region of the active $\mathrm{ZnSe}$ layer are obtained, as revealed by scanning electron microscopy. A high photoluminescence quantum efficiency is observed even in the narrow structures, implying a slow surface recombination rate. In addition to the QWR's unpatterned $100 \times 100 \mu \mathrm{m}^{2}$ mesa structures are provided on the sample as a quasi-2D reference. A detailed description of the fabrication process is given in Ref. 23.

As excitation source for the experiments we used a frequency-doubled mode-locked Ti-sapphire laser, providing $100 \mathrm{fs}$ pulses with a spectral width of $22 \mathrm{meV}$ at a repetition rate of $76 \mathrm{MHz}$. The two-pulse degenerate FWM experiments were performed in reflection geometry, applying collinear polarized input fields. The $1 / e^{2}$ focus diameter of the pulses on the sample was measured with a charge-coupled device camera to $60 \mu \mathrm{m}$. The density dependence was investigated by a variation of the excitation intensity. The FWM signal was recorded time integrated and spectrally resolved by a combination of a spectrometer and an optical multichannel analyzer, as a function of the time delay $\tau$ between the two incident pulses. The samples were kept in a helium cryostat at a temperature of $5 \mathrm{~K}$.

Measurements were made on the QWR arrays, on the mesa structures, and on the unpatterned SQW sample. The normalized FWM spectra at zero delay time are shown in Fig. 1 for the SQW, a mesa structure, and QWR arrays of 132, 46, and $23 \mathrm{~nm}$ wire width. In all cases, the excitation intensity was $280 \mathrm{~kW} / \mathrm{cm}^{2}$ (corresponding to a $2 \mathrm{D}$ exciton density of $1 \times 10^{9} \mathrm{~cm}^{-2}$ ), and the laser center wavelength was set to the lowest excitonic transition, avoiding excitation 


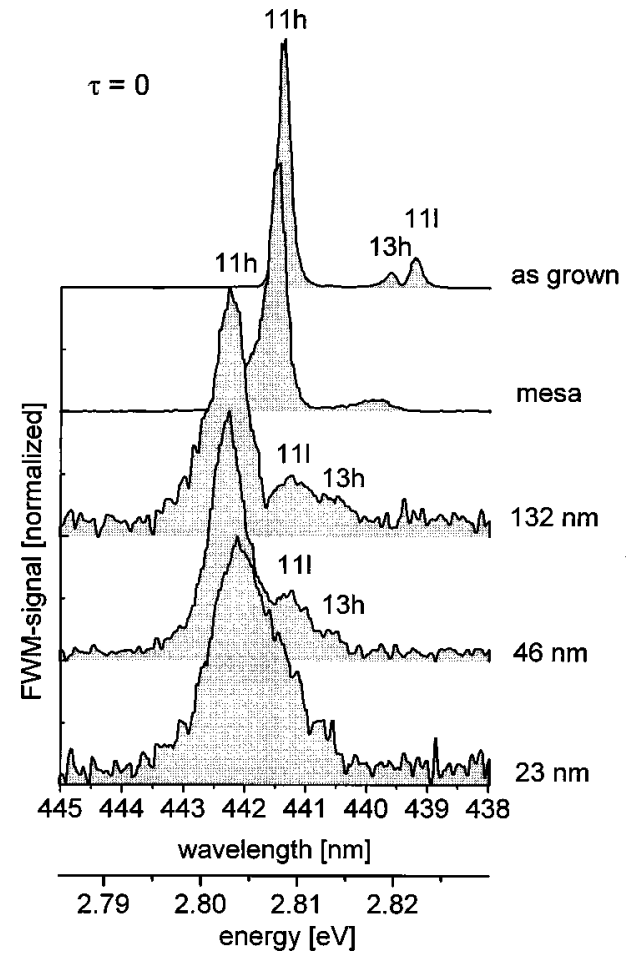

FIG. 1. FWM spectra at zero delay time of the as-grown QW, the QW mesa, and QWR's of 132, 46, and $23 \mathrm{~nm}$ width.

of the excitonic continuum. In the SQW, the compressive biaxial strain and the quantum confinement lift the degeneracy between the heavy hole $(11 h, 13 h)$ and light hole $(11 l)$ $1 s$ exciton states, which are identified by comparison with calculated exciton energies using the model of Mathieu, Lefebvre, and Christol. ${ }^{30}$ In the etched wires, the structure undergoes an elastic relaxation perpendicular to the wire $(\langle 011\rangle)$ direction due to the free-standing sidewalls. This leads to a redshift of the exciton energies in the QWR structures. $^{31,32}$ The strain-induced redshift of the light-hole exciton is stronger than that of the heavy-hole excitons, ${ }^{33}$ which show equal strain shifts. Even though the QWR excitons are not of pure heavy-hole or light-hole character due to the broken rotational symmetry along the growth direction, we denote them after their main component. In the investigated structures, both the SQW width and the wire width are larger than the bulk $\mathrm{ZnSe}$ exciton diameter $(8.6 \mathrm{~nm})$, and even for the smallest investigated wire width of $23 \mathrm{~nm}$, the lateral confinement energy is less than the strain-induced effects. The exciton assignments given in Fig. 1 are made according to these considerations, and are supported by systematic investigations on similar SQW structures. ${ }^{28}$

A small redshift of the transitions already occurs going from the unpatterned SQW to the mesa structure. This is not well understood, but is probably due to surface alterations by the patterning process, influencing the SQW states by surface fields. The 132-nm QWR structure shows an inhomogeneous broadening of about $2 \mathrm{meV}$ due to strain fluctuations or surface fields. The same holds for the $46 \mathrm{~nm}$ QWR structure, showing an equal broadening. Here, the strain shift is essentially saturated, e.g., the structure is relaxed in the $\langle 01 \overline{1}\rangle$ direction. In the $23 \mathrm{~nm}$ QWR, a quantization-induced blueshift of the $11 \mathrm{~h}$ transition is observed. Taking into account the strain shift, the extracted lateral confinement energy is esti-

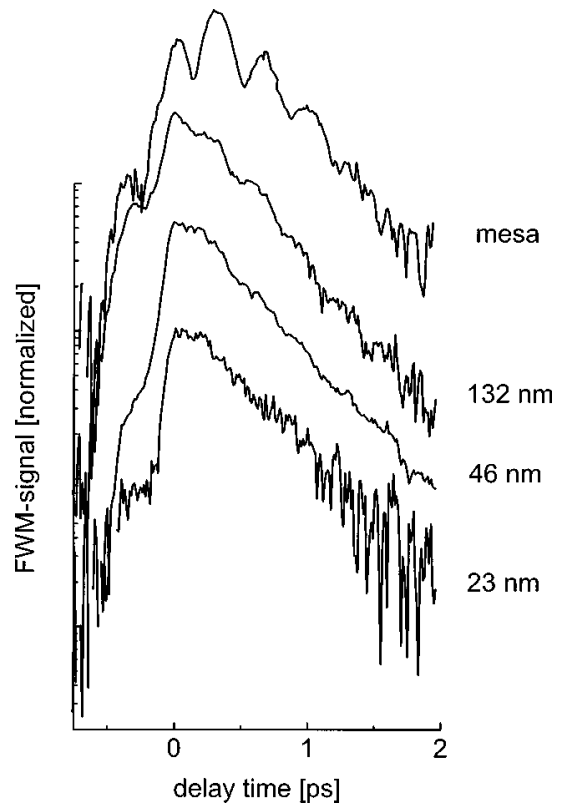

FIG. 2. FWM signal at the energetic position of the $11 \mathrm{~h}$ exciton transition vs delay time for the QW mesa and the QWR's of 132, 46 , and $23 \mathrm{~nm}$ width.

mated to be $2.5 \mathrm{meV}$, in agreement with effective-mass calculations and investigations on $\mathrm{Cd}_{x} \mathrm{Zn}_{1-x} \mathrm{Se} / \mathrm{ZnSe}$ quantum wires. ${ }^{23}$ The lateral size fluctuations due to the fabrication process can be estimated to about $\pm 8 \mathrm{~nm}$ from scanning electron microscopy data, which results in an inhomogeneous broadening of the transitions with decreasing wire sizes due to the fluctuation of the lateral quantization, e.g., in the $23 \mathrm{~nm}$ QWR to a $11 \mathrm{~h}$ transition broadening of about 4 $\mathrm{meV}$. In addition, nonresolved higher quantum wire states join at the high-energy side of the fundamental $11 h, 13 h$, and $11 l$ transitions.

The FWM signal for the different samples, recorded at the $11 \mathrm{~h}$ transition energy as a function of delay time, is shown in Fig. 2. The coherent excitation of the $11 h, 13 h$, and $11 l$ excitons leads to quantum beats in the FWM trace, which become weaker with decreasing wire size. The Fourier transformed FWM traces, shown in Fig. 3, show the energies of the higher transitions relative to the $11 \mathrm{~h}$ exciton. They are in agreement with the energy splittings observed in the FWM spectra (Fig. 1).

The 132-nm QWR shows a slightly higher FWM signal decay rate $\gamma_{d}=2.5 \mathrm{ps}^{-1}$ compared to the mesa ( $\gamma_{d}$ $=2.2 \mathrm{ps}^{-1}$ ) and to the as-grown layer $\left[\gamma_{d}=2.5 \mathrm{ps}^{-1}\right.$ (Ref. 13)]. The small decrease of the decay rate in the QWR structure is attributed to the introduction of sidewall defects and small inhomogeneous broadening due to nonuniform distortion across the wire structure. The 46- and 23-nm QWR's show a decay rate of $\gamma_{d}=2 \mathrm{ps}^{-1}$, which is even lower than the value of the as-grown layer structure. Taking into account inhomogeneous broadening of the system, the deduced dephasing time amounts to $T_{2}=2 \mathrm{ps}$, which is more than twice the value obtained from the mainly homogeneously broadened mesa structure.

Despite the localization of excitons we find a clear monoexponential decay and no redshift of the FWM signal maximum with increasing delay time, showing that the spec- 


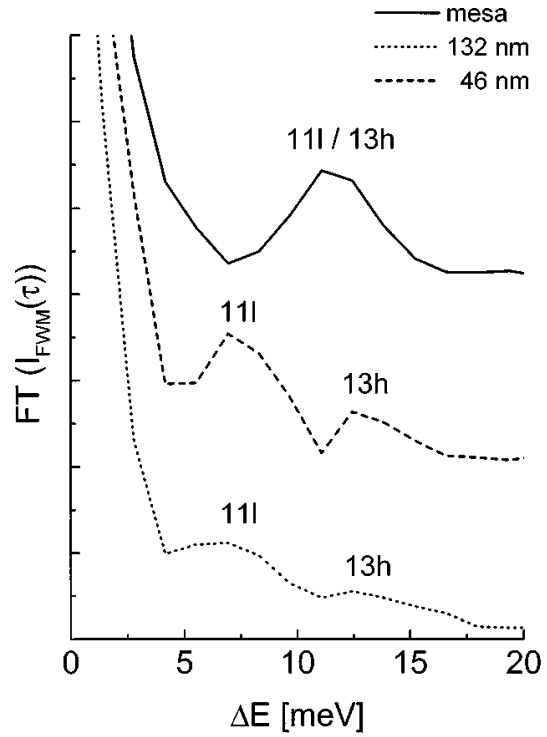

FIG. 3. Fourier transformed FWM traces of Fig. 2 for the QW mesa and the QWR's of 132 and $46 \mathrm{~nm}$ width.

tral relaxation time is long compared to the dephasing time. This feature is not observed in QW structures and might be explained by the reduced acoustic-phonon scattering with localized excitons in a 1D system.

Recent FWM experiments on unpatterned SQW's indicate the presence of free electrons within the well. ${ }^{34}$ These electrons originate from barrier impurities, which are excited by the pulses into the conduction band and captured into the QW. Here the free electrons act as charged scattering particles leading to a constant background dephasing. The increase of the dephasing time $T_{2}$ in the narrow QWR structures might be therefore related to a reduction of the electron-exciton scattering by exciton localization or electron trapping in surface states generated by the wire fabrication process.

Furthermore, the reduction of the coherence volume of the 1D confined excitons leads to a decrease of the excitonphoton coupling. ${ }^{35}$ However, the radiative lifetime of the $11 h$ transition in the SQW can be estimated to about $3 \mathrm{ps},{ }^{36}$ and is thus not the predominant dephasing mechanism even in the unpatterned structure. The influence of the decreased exciton-phonon scattering due to the reduced dimensionality, predicted in Refs. 14 and 18, is estimated to be insignificant for the observed increase of the dephasing time.

The exciton-exciton scattering rate and the low-density dephasing times are determined using the density dependence of the FWM decay, varying the intensity of the two FWM pulses equally. The obtained decay rates are plotted in Fig. 4 as a function of the total incident pulse intensity. The decay rates $\gamma_{d}$ strongly increase with decreasing QWR size. Up to intensities of $200 \mathrm{~nJ} / \mathrm{cm}^{2}$, we observe a linear increase of $\gamma_{d}$ with increasing pulse intensity in all QWR structures. At higher intensities, we find a deviation from this behavior indicating the crossover from an inhomogeneously broadened system, giving a photon echo response, to a mainly homogeneously broadened system, showing a free induction decay. This crossover appears at increasing intensities with decreasing QWR sizes, due to the different inhomogeneous broadening, and is most distinctly observed in the $132 \mathrm{~nm}$

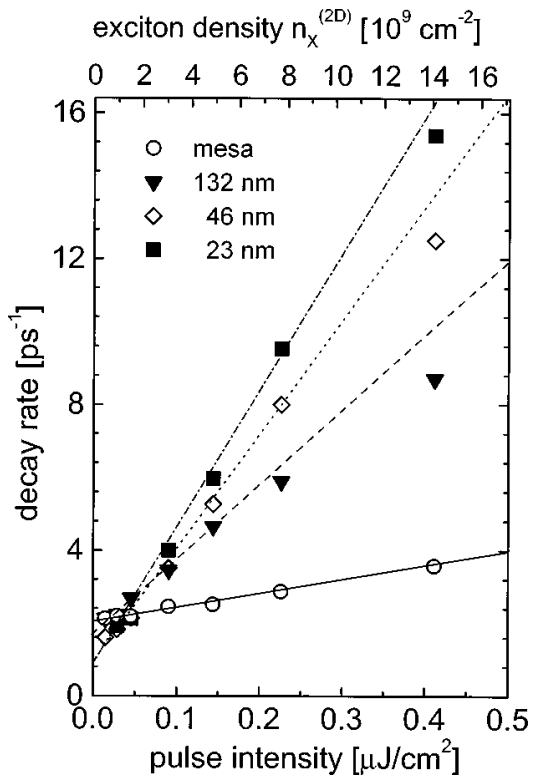

FIG. 4. Decay rates as a function of the total incident pulse intensities for the QW mesa and the QWR's. The dashed curves are guides for the eye. The top axis gives the $2 \mathrm{D}$ exciton density obtained from the QW structure.

QWR. There is no deviation found in the mesa structure, which is a mainly homogeneously broadened system. The homogeneous linewidths $\Gamma_{\text {hom }}=h /\left(\pi T_{2}\right)$ of the QWR excitons can be deduced for intensities below $200 \mathrm{~nJ} / \mathrm{cm}^{2}$ in the inhomogeneous broadening limit from the decay time $T_{d}$ by $T_{2}=4 T_{d}$. The linear increase of $\Gamma_{\text {hom }}$ with increasing exciton density is described by

$$
\Gamma_{\text {hom }}\left(n_{x}\right)=\Gamma_{0}+\beta_{X X}^{(m)} n_{X}^{(m)} .
$$

Here, $\beta_{X X}^{(m)}$ is the exciton-exciton scattering parameter of dimensionality $m$ and $n_{X}^{(m)}$ is the 2D or 1D exciton density, respectively, while $\Gamma_{0}$ is the low-density homogeneous line width. The 2D exciton density (see Fig. 4 top axis) is estimated using the measured SQW absorption, and the measured excitation diameter and intensity. ${ }^{13}$ In calculating the exciton densities in the wire structures, we take alterations of the absorption due to the wire structure into account. As already mentioned, the width of the investigated QWR's exceed the exciton Bohr diameter considerably, so that no significant change of the electron-hole overlap is expected. All experiments were performed with linear polarizations parallel to the wire, giving an increase of the FWM signal by about one order of magnitude compared to the linear polarization perpendicular to the wires. The excitonic oscillator strengths for the two polarizations thus differ by approximately a factor of 1.8. Assuming a conservation of the total oscillator strength, we estimate a 30\% increase of the QWR absorption in the parallel configuration compared to the SQW, which is considered in the determination of the exciton density. The obtained values for $\Gamma_{0}$ and $\beta_{X X}^{(2 \mathrm{D})}$ of the different samples are given in Table I. We observe a clear increase of $\beta_{X X}^{(2 \mathrm{D})}$ with decreasing QWR size. This is attributed to the reduced phase space of the QWR's, causing an increased repulsive interaction between excitons due to the Pauli exclusion principle. For the $23 \mathrm{~nm}$ wire, the use of a 
TABLE I. Low density dephasing times $\Gamma_{0}$ and exciton-exciton scattering parameters $\beta_{x x}^{(2 \mathrm{D})}$ of the investigated samples.

\begin{tabular}{lcc}
\hline \hline Wire width $(\mathrm{nm})$ & $\Gamma_{0}(\mathrm{meV})$ & $\beta_{x x}^{(2 \mathrm{D})}\left(10^{-10} \mathrm{meV} \mathrm{cm}^{2}\right)$ \\
\hline$\infty$ & $1.3 \pm 0.2$ & $0.7 \pm 0.2$ \\
132 & $0.6 \pm 0.2$ & $1.6 \pm 0.3$ \\
46 & $0.3 \pm 0.2$ & $2.5 \pm 0.5$ \\
23 & $0.2 \pm 0.2$ & $3.2 \pm 0.8$ \\
\hline \hline
\end{tabular}

1D exciton density seems more appropriate, since the level spacing supersedes the inhomogeneous broadening. We estimate the $1 \mathrm{D}$ density by the relation $n_{X}^{(1 \mathrm{D})} \approx n_{X}^{(2 \mathrm{D})} \times W$, where $W$ is the wire width. Using Eq. (1), we deduce a $1 \mathrm{D}$ scattering parameter of $\beta_{X X}^{(1 \mathrm{D})}=(1.4 \pm 0.4) \times 10^{-4} \mathrm{meV} \mathrm{cm}$ for the $23 \mathrm{~nm}$ QWR, which is close to the value of $\beta_{X X}^{(1 \mathrm{D})}=1.2 \times 10^{-4} \mathrm{meV} \mathrm{cm}$ found in GaAs QWR's. ${ }^{22}$ The extrapolated homogeneous linewidth $\Gamma_{0}$ is given by the remaining background scattering with free electrons, phonons, and photons (radiative damping). It shows a decrease with decreasing QWR size as already attributed to a reduced exciton-electron scattering due to localization and electron trapping.

We thank T. Reisinger and W. Gebhardt for providing the $\mathrm{Zn}_{0.94} \mathrm{Mg}_{0.06} \mathrm{Se} / \mathrm{ZnSe}$ single $\mathrm{QW}$ structure. This work was supported by the Deutsche Forschungsgemeinschaft and by the Danish Ministries of Research and Education in the framework of the Center for Nanostructures.
${ }^{1}$ L. Schultheis, J. Kuhl, A. Honold, and C. W. Tu, Phys. Rev. Lett. 57, 1635 (1986).

${ }^{2}$ A. Honold, L. Schultheis, J. Kuhl, and C. W. Tu, Phys. Rev. B 40, 6442 (1989).

${ }^{3}$ E. O. Göbel, K. Leo, T. C. Damen, J. Shah, S. Schmitt-Rink, W. Schäfer, J. F. Müller, and K. Köhler, Phys. Rev. Lett. 64, 1801 (1990).

${ }^{4}$ K. Leo, T. C. Damen, J. Shah, and K. Köhler, Phys. Rev. B 42, 11359 (1990).

${ }^{5}$ M. Wegener, D. S. Chemla, S. Schmitt-Rink, and W. Schäfer, Phys. Rev. A 42, 5675 (1990).

${ }^{6}$ A. E. Paul, J. A. Bolger, A. L. Smirl, and J. G. Pellegrino, J. Opt. Soc. Am. B 13, 1016 (1996).

${ }^{7}$ J. Erland, K.-H. Pantke, V. Mizeikis, V. G. Lyssenko, and J. M. Hvam, Phys. Rev. B 50, 15047 (1994).

${ }^{8}$ C. Dörnfeld and J. M. Hvam, IEEE J. Quantum Electron. 25, 904 (1989).

${ }^{9}$ H. Schwab et al., Phys. Status Solidi B 172, 479 (1992).

${ }^{10}$ V. G. Lyssenko, J. Erland, I. Balslev, K.-H. Pantke, B. Razbirin, and J. M. Hvam, Phys. Rev. B 48, 5720 (1993).

${ }^{11}$ A. J. Fischer, D. S. Kim, J. Hays, W. Shan, J. J. Song, D. B. Eason, J. Ren, J. F. Schetzina, H. Luo, J. K. Furdyna, Z. Q. Zhu, T. Yao, J. F. Kiem, and W. Schäfer, Phys. Rev. Lett. 73, 2368 (1994).

${ }^{12}$ T. Häupl, H. Nickolaus, F. Henneberger, and A. Schüzgen, Phys. Status Solidi B 194, 219 (1996).

${ }^{13}$ H. P. Wagner, A. Schätz, R. Meier, W. Langbein, and J. M. Hvam, preceding paper, Phys. Rev. B 57, 1791 (1997).

${ }^{14}$ U. Bockelmann and G. Bastard, Phys. Rev. B 42, 8947 (1990).

${ }^{15}$ R. Cincolani, H. Lage, L. Tapfer, H. Kalt, D. Heitmann, and K. Plog, Phys. Rev. Lett. 67, 891 (1991).

${ }^{16}$ G. Mayer, F. E. Prins, G. Lehr, H. Schweizer, H. Leier, B. E. Maile, J. Straka, A. Forchel, and G. Weimann, Phys. Rev. B 47, 4060 (1993).

${ }^{17}$ G. Fasol and H. Sakaki, Phys. Rev. Lett. 70, 3643 (1993).

${ }^{18}$ U. Bockelmann, Semicond. Sci. Technol. 9, 865 (1994).

${ }^{19}$ E. Kapon, D. W. Hwang, and R. Bhat, Phys. Rev. Lett. 63, 430 (1989).
${ }^{20}$ P. Ils, M. Michel, A. Forchel, I. Gyoro, M. Klenk, and E. Zielinsky, Appl. Phys. Lett. 64, 496 (1994).

${ }^{21}$ Y. Naganume, Y. Arakawa, M. Nishioka, and S. Tsukamoto, Semicond. Sci. Technol. 8, 1082 (1993).

${ }^{22}$ E. J. Mayer, J. O. White, G. O. Smith, H. Lage, D. Heitmann, K. Ploog, and J. Kuhl, Phys. Rev. B 49, 2993 (1994).

${ }^{23}$ M. Illing, G. Bacher, T. Kümmel, A. Forchel, T. G. Anderson, D. Hommel, D. Jobst, and G. Landwehr, Appl. Phys. Lett. 67, 124 (1995).

${ }^{24}$ G. Bacher, M. Illing, R. Spiegel, T. Kümmel, K. Herz, A. Forchel, B. Jobst, D. Hommel, and G. Landwehr, J. Cryst. Growth 159, 455 (1996).

${ }^{25}$ T. Saiki, K. Takeuchi, and M. Kuwata-Gonokamie, Appl. Phys. Lett. 60, 192 (1992).

${ }^{26}$ A. Knorr, T. Stroucken, A. Schulze, A. Girndt, and S. W. Koch, Phys. Status Solidi B 188, 473 (1995).

${ }^{27}$ M. Hubner, J. Kuhl, J. Stroucken, A. Knorr, S. W. Koch, R. Hey, and K. Ploog, Phys. Rev. Lett. 76, 4199 (1996).

${ }^{28}$ T. Reisinger, Ph. D. thesis, Universität Regensburg, Germany, 1996.

${ }^{29}$ M. Wörz, E. Griebl, Th. Reisinger, B. Flierl, D. Haserer, T. Semmler, T. Frey, and W. Gebhardt, Phys. Status Solid B 202, 805 (1997).

${ }^{30}$ H. Mathieu, P. Lefebvre, and P. Christol, Phys. Rev. B 46, 4092 (1992).

${ }^{31}$ I.-H. Tan, R. Mirin, T. Yasuda, E. L. Hu, J. Bowers, C. B. Prater, P. K. Hansma, M. Y. He, and A. G. Evans, J. Vac. Sci. Technol. B 10, 1971 (1992).

${ }^{32}$ G. Lermann, T. Bischof, A. Materny, W. Kiefer, T. Kümmel, G. Bacher, A. Forchel, and G. Landwehr, J. Appl. Phys. 81, 1446 (1997).

${ }^{33}$ H. Straub, G. Brunthaler, W. Faschinger, and G. Bauer, Acta Phys. Pol. A 90, 1085 (1996).

${ }^{34}$ H. P. Wagner, A. Schätz, R. Meier, W. Langbein, and J. M. Hvam (unpublished).

${ }^{35}$ D. S. Citrin, Phys. Rev. Lett. 69, 3393 (1992).

${ }^{36}$ S. Jorda, U. Rössler, and D. Broido, Phys. Rev. B 48, 1669 (1993). 